\title{
Ring Patterns - distribution, diversification or speciation? A case study of two small mammals in the mountains of Southwest China
}

\author{
Yanqun Wang ${ }^{1}$, Anderson Feijó ${ }^{1}$, Jilong Cheng ${ }^{1}$, Lin Xia ${ }^{1}$, Zhixin Wen ${ }^{2}$, Deyan $\mathrm{Ge}^{3}$, Jian \\ Sun ${ }^{1}$, Liang $\mathrm{Lu}^{4}$, Song $\mathrm{Li}^{5}$, and Qisen Yang ${ }^{1}$ \\ ${ }^{1}$ Institute of Zoology Chinese Academy of Sciences \\ ${ }^{2}$ Key Laboratory of Zoological Systematics and Evolution, Institute of Zoology, Chinese \\ Academy of Sciences, Beichen West Road, Chaoyang District, Beijing 100101 \\ ${ }^{3}$ Key Laboratory of Zoological Systematics and Evolution, Institute of Zoology, Chinese \\ Academy of Sciences, Beichen West Road, Chaoyang District, Beijing 100101,China \\ ${ }^{4}$ National Institute for Communicable Disease Control and Prevention, China CDC \\ ${ }^{5}$ Kunming Institute of Zoology Chinese Academy of Sciences
}

April 28, 2020

\begin{abstract}
Ring species and ring diversification provide good evidence of gradual speciation. Studying differentiation in ring species/ring diversification patterns contributes to understanding the process of speciation. We applied a fine-scale phylogeographic survey to two congeneric small mammals, the South China field mouse (Apodemus draco) and the Chevrier's field mouse (A. chevrieri), which are endemic to the mountains of Southwest China (MSC), combining mitochondrial (Cytb and COI) and nuclear (microsatellite loci) markers, with dense sampling throughout the range $(411 \mathrm{~A}$. draco from 21 sites and 191 A. chevrieri from 22 sites), as well as species distribution modeling, scenario testing of dispersal routes and redundancy analyses of environmental and spatial factors to characterize the population genetic structure and infer the proximate formation mechanism of these patterns. Our results revealed that both A. draco and A. chevrieri clustered into western and eastern lineages, which dispersed clockwise and anticlockwise, respectively, from west to northwest. The two species showed gradually increasing genetic differences with geographic distance and displayed a ring diversification pattern around the Sichuan Basin. The tectonic events of the QinghaiTibetan Plateau (QTP) and climatic oscillations during the Quaternary triggered the genetic divergence of A. draco and A. chevrieri by providing environmental heterogeneity and spatial variation, and shaped the ring diversification pattern with the effect of species traits. Our report on the two ring patterns provides supportive evidence for the hypothesis that speciation is a continuous process in nature. Key words: small mammal, phylogeography, speciation, ring species, diversification, mountains of Southwest China
\end{abstract}

Ring Patterns - dist ribution, diversification or speciation? A case study of two small mammals in the mountains ofSouthwest China

running title: Two ring diversification patterns

Yanqun Wang ${ }^{1,2,3}$, Anderson Feijó ${ }^{1}$, Jilong Cheng ${ }^{1}$, Lin Xia ${ }^{1}$, Zhixin Wen ${ }^{1}$, Deyan $\mathrm{Ge}^{1}$, Jian Sun ${ }^{1}$, Liang $\mathrm{Lu}^{4}$, Song $\mathrm{Li}^{5}$, Qisen Yang ${ }^{1 *}$

1 Key Laboratory of Zoological Systematics and Evolution, Institute of Zoology, Chinese Academy of Sciences, Beijing 100101, China

2 Key Laboratory of Animal Disease Detection and Prevention in Panxi District, Xichang College, Xichang, 415000, Sichuan Province, China 
3 University of Chinese Academy of Sciences, Beijing 100049, China

4 State Key Laboratory for Infectious Disease Prevention and Control, National Institute for Communicable Disease Control and Prevention, Chinese Centre for Disease Control and Prevention, Beijing 102206, China

5 Kunming Institute of Zoology, Chinese Academy of Sciences, Kunming, Yunnan 650223, China

* Corresponding author:

Qisen Yang, Key Laboratory of Zoological Systematics and Evolution, Institute of Zoology, Chinese Academy of Sciences, Beijing 100101, China

Email: yangqs@ioz.ac.cn

\begin{abstract}
Ring species and ring diversification provide good evidence of gradual speciation. Studying differentiation in ring species/ring diversification patterns contributes to understanding the process of speciation. We applied a fine-scale phylogeographic survey to two congeneric small mammals, the South China field mouse (Apodemus draco ) and the Chevrier's field mouse (A. chevrieri), which are endemic to the mountains of Southwest China (MSC), combining mitochondrial ( $C y t b$ and $C O I$ ) and nuclear (microsatellite loci) markers, with dense sampling throughout the range (411 A. draco from 21 sites and $191 \mathrm{~A}$. chevrieri from 22 sites), as well as species distribution modeling, scenario testing of dispersal routes and redundancy analyses of environmental and spatial factors to characterize the population genetic structure and infer the proximate formation mechanism of these patterns. Our results revealed that both $A$. draco and A. chevriericlustered into western and eastern lineages, which dispersed clockwise and anticlockwise, respectively, from west to northwest. The two species showed gradually increasing genetic differences with geographic distance and displayed a ring diversification pattern around the Sichuan Basin. The tectonic events of the Qinghai-Tibetan Plateau (QTP) and climatic oscillations during the Quaternary triggered the genetic divergence of $A$. draco and A. chevrieri by providing environmental heterogeneity and spatial variation, and shaped the ring diversification pattern with the effect of species traits. Our report on the two ring patterns provides supportive evidence for the hypothesis that speciation is a continuous process in nature.
\end{abstract}

Key words: small mammal, phylogeography, speciation, ring species, diversification, mountains of Southwest China

\title{
Introduction
}

The process of speciation has long fascinated evolutionary biologists, and the core of microevolutionary research is the understanding of species differentiation during speciation (Foster, Scott, \& Cresko, 1998; Rice \& Hostert, 1993). Ring species are recognized to be good evidence of gradual speciation in nature, in which a chain of intergrading populations encircles a barrier and terminal forms coexist without interbreeding, providing a situation in which variation in space can be used to infer variation in time (Irwin, Irwin, \& Price, 2001; Mayr, 1942). Previous studies of the greenish warbler Phylloscopus trochiloides complex ring around the Tibetan Plateau (Irwin, Bensch, \& Price, 2001) and the Ensatinaeschscholtzii complex ring in central coastal California (Kuchta, Parks, \& Wake, 2009) have well explained how geographic variation leads to speciation, even in the presence of gene flow.

In recent years, some new cases of 'ring species' have been reported, such as theAlophoixusbulbuls complex around the Thailand lowlands (Fuchs, Ericson, Bonillo, Couloux, \& Pasquet, 2015) andPlatycercus elegans complex in eastern Australia (Joseph et al., 2008). These species complexes show a ring distribution and hierarchical genetic pattern surrounding a barrier without evidence of reproductive isolation at the terminals of the ring. Even in some classic ring species cases, it is difficult to encounter strict reproductive isolation, because gene flow resumes again after secondary contact (Alcaide, Scordato, Price, \& Irwin, 2014; Devitt, Baird, \& Moritz, 2011; Pereira \& Wake, 2015), and the identification of full reproductive isolation (valid species) remains a difficult task for taxonomists (Liebers, Knijff, \& Helbig, 2004). Thus, Monahan (2012) used 'ring diversification' rather than 'ring species' to describe the situation in which populations diverge 
around a geographic barrier in a ring-like manner, regardless of whether reproductive isolation occurs at the terminus, such as for the eastern tree frog (Hyla orientalis ) around the Black Sea (Dufresnes et al., 2016). Compared with 'ring species', 'ring diversification' is far less restrictive, and can be extended to more natural cases to explore the process of speciation as well as the mechanism of species formation (Petit et al., 2003). However, until now, birds and amphibians have been the main target for investigating ring species/ring diversification. To our knowledge, no mammal has been described as a ring species, although an indispensable part of the earth ecosystem.

Undoubtedly, ring-shaped divergence is based on a ring-shaped distribution, but not all taxa inhabiting a ring represent ring diversification or ring species. The genetic structure of the Yarkand hare (Lepus yarkandensis ), which is distributed around the Taklamakan Desert in China, showed no relationship with increasing geographic distance (Wu, Xia, Zhang, \& Yang, 2010). Another example is the herring gull (Larus argentatus ) complex, which comprises more than 20 taxa that occupy a circumpolar breeding range in the Northern Hemisphere. This complex was initially envisioned to be a ring species that had reached full reproductive isolation and coexist as distinct species. However, a later study based on molecular evidence showed that it had only a ring distribution without any ring diversification, let alone a ring species (Liebers et al., 2004). The ring distribution pattern with the potential to form ring diversification or ring species commonly has a specific ring topography: inhospitable habitat in the center of the ring persistently exists such that mating is constrained by spatial and genetic distance, and the geographic structure around the barrier supports a stably suitable habitat for the candidate taxa (Monahan et al., 2012).

The mountains of Southwest China (MSC) represent a global biodiversity hot-spot with typical mountainous landscape features (high mountains which are cut by deep valleys and tumbling rivers), and they have played an important role in the diversification process and genetic patterns of organisms in this region (Chen, He, Cheng, Khanal, \& Jiang, 2017; Lv et al., 2018; Myers, Mittermeier, Mittermeier, \& Kent, 2000; Wan, He, Jin, Liu, \& Jiang, 2018). The MSC (with the exception of the Himalaya Mountains and the southern part of the Hengduan Mountains (HDMs)) forms an approximate ring surrounding the Sichuan Basin, which is mainly a plain with an elevational range of 250-750 m (Liu, Deng, Li, \& Wei, 2012). The great difference in elevation leads to landscape heterogeneity between the basin and the MSC, resulting in a 'soft' barrier to the dispersal of some species (Bennett \& Provan, 2008; He et al., 2019). Especially for the montane species living in the MSC, the basin has become a dispersal barrier (Qiao et al., 2018). The barrier effect of the Sichuan Basin was inferred by Monahan (2012) through developing a generalized model and using the known examples of ring species to evaluate the potential geographic barriers.

Compared with birds and amphibians, small mammals have relatively moderate mobility, and they are characterized by a short generation time. These features make them good candidates for exploring the underlying mechanism of how geographic distribution patterns affect genetic variation. The South China field mouse ( Apodemus draco ) and the Chevrier's field mouse ( A. chevrieri ) are two congeneric small mammals which are commonly distributed in temperate forests at elevations of 1,000-3,500 m around the Sichuan Basin, and they share many distributional ranges (A.draco has a larger distributional range thanA.chevrieri as it also occurs in the mountains of southern China, such as Wuyi mountain in Fujian and Tianmu mountain in Zhejiang) and life history attributes (Fan et al., 2012; Liu et al., 2018; Liu, Wei, Li, \& Feng, 2002). Previous studies have revealed that $A$. draco and $A$. chevrieri diverged from their sister species approximately 2.5-3 Mya. The HDMs were considered the Pleistocene refugia or the radiation center of the two species (Ge et al., 2019; Liu et al., 2012). Intraspecific genetic variation of the two species in this area has been documented, and significant differences in skull morphology of A. chevrieri among populations in parts of the HDMs also showed a positive correlation with geographical distance (Fan et al., 2012; Sakka et al., 2010; Yue et al., 2012; Zhang, Zhang, Mu, Huang, \& Hu, 2008; Zhu, Jiang, Zhang, \& Wang, 2014). These two species are good models for studies on genetic diversification focusing on the influence of combined geography and climatic change because both species were affected by the Quaternary glacial and interglacial cycles during divergence, and the two species could buffer the impact of climate oscillations through the effective use of topographic features. In addition, taking two species as subjects also helps to distinguish the effects of external factors and species traits on diversification. 
Here, we examined the pattern of the genetic diversification of $A$. dracoand A. chevrieri, and inferred the formation mechanism of these patterns by restructuring their demographic history and characterizing their population genetic structure, combining mitochondrial ( $C y t b$ and $C O I$ ) and nuclear (microsatellite loci) markers with dense sampling through this range. We hypothesize that (a) bothA. draco and A. chevrieri diversified in a ring-like manner in view of their species traits 'fitting' the special geographic condition of the barrier (Sichuan Basin) and the existence of the favorable conditions for divergence. (b) However, full reproductive isolation at the terminals of the two rings was not observed because the size of the barrier was not sufficiently large to form a valid species.

\section{Materials and Methods}

$\mathrm{S}$ ample collec tion

A total of $411 \mathrm{~A}$. draco individuals from 21 sites and $191 \mathrm{~A}$. chevrieriindividuals from 22 sites at elevations of 1,360-3,370 m were sampled, covering most of their distributional ranges around the Sichuan Basin. Detailed information about the sampling site and sample size are provided in Appendix S1. All experimental protocols were approved by the Animal Care and Use Committee of the Institute of Zoology, Chinese Academy of Sciences.

\section{Laboratory protocols}

DNA samples were obtained from ethanol-preserved tissues, and extracted using the DNeasy Tissue Kit (Qiagen, Beijing, China), and vouchers were deposited at the Institute of Zoology, Chinese Academy of Sciences, Beijing, China.

Two mitochondrial markers cytochrome b ( Cytb ) and cytochrome oxidase subunit I (COI), were targeted for PCR and sequencing. The $C y t b$ and $C O I$ genes have demonstrated high levels of variability that are suitable for intraspecific comparison (Fan et al., 2012; Suzuki et al., 2015). We used the universal primers of mammalianCytb L14724/H15915 and COI VF1/VR1 for amplification (Irwin, Kocher, \& Wilson, 1991; Li et al., 2015). A standard PCR protocol was used with an annealing temperature of $53^{\circ} \mathrm{C}$ for $C y t b$ and $55^{\circ} \mathrm{C}$ for $C O I$. The same primers were used for amplification on an ABI 3730XL sequencer (ABI) (Applied Biosystems, Foster City, California, USA). Sequences were assembled with SeqMan (DNASTAR, Lasergene v7.1) before subsequent analyses and aligned using the TranslatorX server online (http://translatorx.co.uk/?COLLCC=276893672783). Conserved blocks were identified using Gblocks 0.91b online (http://www.phylogeny.fr/one_task.cgi?task_type=gblocks) with a more stringent selection.

Eight microsatellite DNA loci were genotyped each for $A$. draco and $A$. chevrieri as described in Gu et al. (2009) and Wu et al. (2009). Information on the primers is provided in Table S1. The primers were labeled with HEX, FAM or TAMRA fluorescence dyes. The PCR protocols followed the previous studies (Gu et al., 2009; Wu et al., 2009). All reactions were conducted separately with one pair of primers. Then, PCR products with different dyes were pooled and genotyped with an ABI 3730XL sequencer. Microsatellite genotypic data were analyzed with GeneMapper4.0 (SoftGenetics, State College, PA, USA).

Phylogenetic analyses and divergence time estimation

The concatenated sequences of the Cytb and COI genes were used for the subsequent mtDNA analyses. We performed maximum-likelihood (ML) and Bayesian inference (BI) phylogenetic reconstructions of the haplotypes of the concatenated sequences with PhyML3.1 (Guindon \& Gascuel, 2003) and MrBayes 3.3.6 (Ronquist et al., 2012). One GTR $+\mathrm{I}+\mathrm{G}$ and one $\mathrm{TVM}+\mathrm{I}+\mathrm{G}$ model of nucleotide substitution were selected for A. draco and A.chevrieri with JModeltest 2.1.7 (Posada \& Crandall, 2001). For the ML analysis, the significance of each node was tested with 1000 bootstrap replicates. For the BI analysis, two independent parallel runs of four incrementally heated Metropolis-coupled Monte Carlo Markov chains (MCMCs) were run for 50 million or more generations, with trees sampled every 1000 generations, until the average standard deviation of the split frequencies was less than $0.01(\mathrm{SD}<0.01)$. The first $25 \%$ of the Markov chain samples were discarded as burn-in, and the chains were checked for stationarity with TRACER 1.7 (Rambaut, Drummond, Xie, 
Baele, \& Suchard, 2018). The final trees were viewed in FigTree 1.4.2 (http://tree.bio.ed.ac.uk/software/figtree/).Apodemus agrarius and $A$. ilex were used for the outgroup species. Previous studies have suggested that the two species are closely related to $A$. draco and A. chevrieri, respectively (Liu et al., 2018), and that A. draco split from A. ilex approximately 3.26 Mya while A. chevrieri split from A. agrarius approximately 2.6 Mya (Ge et al., 2019).

To estimate the divergence time of the main mitochondrial lineages of $A$. draco and A. chevrieri, we performed a Bayesian MCMC analysis in BEAST 1.8.2 (Drummond \& Rambaut, 2007). Two fossil calibration points were used for both species: (a) the basal split time in Murinae (12 Mya), which has frequently been used as a fossil calibration point in mammal studies (Johnson, Stix, Tauxe, Cerveny, \& Tahirkheli, 1985), and (b) a calibration point for the split between Apodemus and Tokudaia (10.5 Mya), based on the recent study by Kimura et al. (2017). The sequences used as outgroups are provided in Table S2. BEAUTI 1.8.2 (in the BEAST 1.82 package) was used to create the XML formatted input files for BEAST. The Hasegawa-KishonoYano (HKY) model of base pair substitution and a strict molecular clock were used. A normal distribution was employed, and default values were used for the other priors. The MCMC chains were analyzed for 200 million generations, with sampling every 2000 generations. TRACER 1.6 was used to verify the posterior distribution and the effective sample sizes (ESSs) from the MCMC output. TreeAnnotator in the BEAST package was used to summarize tree data according to the 'mean height', and the first $25 \%$ of trees were discarded to represent the 'burn-in' period, which ended well after the stationarity of the chain likelihood values had been established. The tree and divergence times are displayed in FigTree 1.4.2.

$\mathrm{P}$ opulation genetic analyses

Null alleles were detected with MICRO-CHECKER 2.2 (Oosterhout, Hutchinson, Wills, \& Shipley, 2004). All descriptive statistics for the microsatellite data, including the average number of alleles per locus and observed and expected heterozygosity, were calculated with GenAlEx 6.51 (Peakall \& Smouse, 2012). The possibility of deviation from Hardy-Weinberg equilibrium (HWE) and linkage equilibrium were tested using Genepop4.2 online (http://www.genepop.curtin.edu.au/) (Raymond, 1995; Rousset, 2008).

We performed a series of analyses to assess the nuclear genetic diversification of A. draco and A. chevrieri around the Sichuan Basin. First, we detected naturally occurring clusters among the individual microsatellite genotypes with STRUCTURE 2.3.4 (Pritchard, Stephens, \& Donnelly, 2000) using the admixture ancestry model. We tested from 1 to 10 groups $(K)$, each group involved 10 replicate runs and consisted of 100,000 iterations after a burn-in period of 10,000 runs. We calculated the average log-likelihood $\operatorname{Pr}(\mathrm{X}-\mathrm{K})$ and the delta-k ad hoc statistics (Evanno, Regnaut, \& Goudet, 2005) with the online program STRUCTURE HARVESTER (http://taylor0.biology.ucla.edu/structureHarvester/) (Earl \& Vonholdt, 2012) to evaluate the number of groups that best explains the data. Second, the population genetic structure was also evaluated with a discriminant analysis of principal components (DAPC) in the R package Adegenet (Jombart, Devillard, \& Balloux, 2010). Pairwise $\mathrm{F}_{\mathrm{ST}}$ values were first estimated using Arlequin 3.5 (Excoffier \& Lischer, 2010), and they were used to represent differences among the populations for the analysis. Third, to evaluate the relationship between genetic distance and geographic distance, the isolation-by-distance (IBD) pattern was examined using GenaAlex 6.5 (Peakall \& Smouse, 2012). To exclude the impact of the small sample size, sites with fewer than 15 individuals were removed from this analysis, except the HeS $(\mathrm{n}=6)$ site for $A$. draco and the GGS $(\mathrm{n}=10)$ and $\mathrm{BaX}(\mathrm{n}=4)$ sites for $A$. chevrieri because they are located in a contact zone. Genetic distance was represented by $\mathrm{F}_{\mathrm{ST}} /\left(1-\mathrm{F}_{\mathrm{ST}}\right)$, and geographic distances were calculated using the coordinate distance calculator online (http://boulter.com/gps/distance/). 'Straight distances' between the sites, which may be located across the uninhabitable basin from one another and adjusted 'ring distances' which assume that dispersal routes followed the ring distribution, were calculated. Based on the results of the mtDNA phylogenetic analyses and nuclear DNA STRUCTURE analyses, we assumed that there is a genetic 'gap' between HeS and WoL for A. draco ; and between GGS and BaX for A. chevrieri. Based on the assumed 'gap', we calculated the ring distances for pairs of sites on the ring with HeS as the starting point of the ring and WoL as the end for $A$. draco and with GGS as the starting point of the ring and BaX as the end for A. chevrieri . A Mantel test implemented in GenaAlex with 9,999 permutations was used to detect a 
significant correlation between the genetic distances and geographic distances using four reference points (A, B, C and D) to approximate the ring distances between sites (Appendix S1). Fourth, to detect the sudden changes in population genetic composition and 'hybrid zones' along the distribution ring, a geographic cline analysis was performed using the R package HZAR (Derryberry, Derryberry, Maley, \& Brumfield, 2014). We first performed a principal component analysis (PCA) of the genetic distance on the microsatellite dataset and used the normalized first principal component as a surrogate for overall genetic distance. Clines were defined by the ring distances. Models with distinct complexities were compared (null model, no exponential tails, one right exponential tail, one left exponential tail, two mirrored exponential tails, and two tails with independent parameters) and ranked by the second-order corrected Akaike information criterion (AICc). In all models, we fixed the minimum and maximum observed mean values. The Markov chain process was set to 1,000,000 in length with a burn-in of 10,000 and an independent seed for each model.

We performed approximate Bayesian computation (ABC) analyses to test the scenarios of dispersal routes for $A$. draco and A. chevrieri distributed around the Sichuan Basin using DIYABC version 2.1 (Cornuet et al., 2014). The scenarios were reconstructed with a step-by-step approach according to two sequential steps of the colonization history, from the ancestral populations to the dispersal routes. To reduce the complexity of the colonization scenario, we considered plausible scenarios based only on the results of the genetic structure analyses in this study and previous studies (Fan et al., 2012; Ge et al., 2019; Yue et al., 2012), and the sampled individuals were treated as four genetic groups, as detected by STRUCTURE (when $K=4$ ), named W1, W2, E1, and E2 (Fig. 3 A1 and B1; Appendix S2). First, we rebuilt three scenario models involving the ancestral population and the two parapatric populations (W2, E1) to describe the dispersal routes of the ancestral population (W1). Second, we tested three and five scenarios for A. dracoandA. chevrieri, respectively, based on the most appropriate scenario in the first step after adding the fourth population E2. Details of the prior parameters for the range of the effective population size $(N e)$, admixture rate $(r)$ between tested populations, and divergence time $(T)$ for the scenarios are described in the Table S3. To assess the model goodness-of-fit for each of the scenario model involving W1, W2, E1, and E2, we performed model checking based on all summary statistics. The results are presented on each plane of the principal component analysis; if the model fits the data well, the observed data (large yellow dot) are observed to be within the scope of simulated datasets of posteriors (solid dots). We then estimated the parameters of the most appropriate scenario in the posterior distribution using a logistic regression.

To understand whether gene flow occurs between parapatric groups, the historical gene flow was detected with the isolation-with-migration model using IMa2p (Hey, 2010; Sethuraman \& Hey). The stepwise-mutationmodel (SSM) was employed for the nuclear microsatellite loci, and the topologies ((W1, W2), (E1, E2)) that represented the most appropriate dispersal scenario for $A$. draco and A. chevrieri (based on the results of the diy ABC analyses) were used. The effective population size $(N e)$, migration rate $(M)$, and effective number of migrants per generation $(2 N M)$ were identified. First, we estimated the functions of the model parameters in the ' $\mathrm{M}$ mode', and generated a burn trend file every three hours until all trends in the plots converged. Then, we ran the 'L mode' to estimate the marginal posterior distribution and the maximumlikelihood estimates of the demographic parameters. The statistical significance of detected gene flow was tested with likelihood-ratio tests.

The relative importance of environmental and spatial factors in the genetic variation of the $A$. draco and A. chevrieri populations was assessed using redundancy analysis (RDA) in the vegan package (Forester, Lasky, Wagner, \& Urban, 2018; Kierepka \& Latch, 2015). All axes of the principal coordinate analysis $(\mathrm{PCoA})$ of the genetic distance matrix were used as dependent variables to summarize the genetic divergence among individuals. According to the DAPC results, we grouped all populations into three regional groups: North (N), Northwest (NW) and West (W) (Appendix S2). The explanatory variables included the spatial coordinates (latitude and longitude), environmental variables (net primary productivity (NPP), potential evapotranspiration (PET), and nineteen bioclimatic parameters for the present time (Table S4)). To avoid collinearity, highly correlated climatic variables $(\mathrm{R}>0.8)$ were removed. We assessed the contributions of isolated and combined sets of explanatory variables (spatial and environmental) by running three models: (a) a model combining environmental and spatial variables as explanatory variables, (b) a model with the 
isolated effect of environmental variables controlling for spatial influence, and (c) a model with the isolated effect of spatial structure controlling for environmental influence.

Species distribution modeling

We used species distribution modeling (SDM) to identify the potential suitable distributional ranges of each species for the present time, the last interglacial (LIG; approximately 130,000 BP) and the last glacial maximum (LGM; approximately 22,000 BP). In addition to the sites sampled for species presence data, we also examined museum collections and checked other sites mentioned in publications (Fan et al., 2012; Liu et al., 2002; Liu et al., 2004; Sakka et al., 2010; Suzuki et al., 2008; Yue et al., 2012). To avoid possible wrong information for the historical records, only sites with verified records were used. Duplicated localities were removed with ENMTools 1.4.4 (Glor \& Warren, 2011; Warren, Glor, \& Turelli). Finally, 33 and 27 sites for $A$. draco and $A$. chevrieri , respectively, were used to build the species distribution modeling (Appendix S1). Nineteen bioclimatic layers for the present time and LIG/LGM (30 sec resolution) were obtained from the WorldClim database (http://worldclim.org/version2) (Table S4) (Fick \& Hijmans, 2017). To prevent redundancy, correlations between pairs of bioclimatic parameters were assessed using Pearson's correlation tests in ENMTools 1.4.4 (Synes \& Osborne, 2011). For each highly correlated variable pair $(|\mathrm{r}|[?] 0.80)$, the variable that gave the highest value in the regularized gain and/or the percent contribution to the MAXENT model was retained. Consequently, nine variables (bio_1 Annual Mean Temperature, bio_2 Mean Diurnal Range, bio_3 Isothermality, bio_4 Temperature Seasonality, bio_7 Temperature Annual Range, bio_12 Annual Precipitation, bio_14 Precipitation of Driest Month, bio_15 Precipitation of Seasonality, and bio_18 Precipitation of Coldest Quarter) were included in the final analyses. Models were constructed with MAXENT version 3.3.3e, using the default settings (Phillips, Anderson, \& Schapire; Provan \& Bennett). The area under the receiver operating characteristic curve (AUC) was used to evaluate the accuracy of our predicted models, and models with AUC values above 0.75 were considered useful, while those with value above 0.90 were considered very good (Elith, 2000; Swets, 1988). An easily interpretable logistic output format was selected with suitability values ranging from 0 (lowest) to 1 (highest) (Phillips et al., 2006). The $\mathrm{SDM}$ analysis was performed at a resolution of 30 -arc seconds $(\sim 1 \mathrm{~km})$.

\section{Results}

Mitochondrial phylogenetic inferences and divergence time estimation

We obtained 1488 bp (Cytb 891 bp; COI 597 bp) and 1611 bp (Cytb 1014 bp; COI 597 bp) concatenated mitochondrial sequences for $A$. draco andA. chevrieri, respectively. The phylogenetic inferences according to both the BI and ML analyses indicated four major evolutionary clades (A. draco: D1, D2, D3, and D4;A. chevrieri : $\mathrm{C} 1, \mathrm{C} 2, \mathrm{C} 3$, and $\mathrm{C} 4)$ with several subdivisions for the two species. For A. draco, the early diverged clade occurred in the west (clade D1), intermediate clades were found in the east and the north (clade D2 and D3; including part of the Yunnan-Guizhou Plateau (YGP), Daba Mountains, Wu Mountains, the south slope of the Qinling Mountains, and part of the eastern HDMs), and recent diversification occurred in the northwest (clade D4, part of the east HDMs) (Fig. 1A and Fig. 2A). For A. chevrieri, early divergence also occurred in the west $(\mathrm{C} 1)$, followed by the $\mathrm{C} 2$ clade in the northwest, and recent diversification occurred between $\mathrm{C} 3$ and $\mathrm{C} 4$ in the north (Fig. 1B and Fig. 2B).

Molecular dating for $A$. draco and A. chevrieri based on the concatenated mitochondrial sequence indicated the occurrence of two main diversification periods. For $A$. draco, clade D1 first diverged from other clades approximately 1.6 Mya (95\% HDPs between 1.3 and 1.9); the south clade split from the other clades approximately 1.3 Mya (95\% HDPs between 1.0 and 1.6); D2 split from the other clades approximately 1.0 Mya (95\% HDPs between 0.8 and 1.3); then, D3 split from D4 approximately 0.58 Mya (95\% HDPs between 0.4 and 0.7) (Fig. 2A). For A. chevrieri, C1 and the other clades first split approximately 1.1 Mya (95\% HDPs between 0.79 and 1.46); C2 split from the other clades approximately 0.78 Mya (95\% HDPs between 0.55 and 1.05); then, C3 split from C4 approximately 0.7 Mya (95\% HDPs between 0.48 and 0.93) (Fig. 2B).

Nuclear genetic differentiation 
A total of $362 \mathrm{~A}$. draco from 21 sites and $191 \mathrm{~A}$. chevrieri from 22 sites were genotyped for the microsatellite DNA loci (Appendices S1 and S2). A few null alleles detected were removed from the analyses. After Bonferroni correction $(\mathrm{p}<0.01)$, the loci did not show significant deviation from $\operatorname{HWE}(\mathrm{p}<0.01)$. Linkage disequilibrium was observed within populations $(\mathrm{p}<0.01)$.

In the STRUCTURE analyses of the two species, the highest likelihoods of the microsatellite genotypes were obtained when samples were clustered into two major lineages (western and eastern lineages) (i.e., $K=2$ ) (Fig. 3 A2 and B2). Further population structure analyses with DAPC supported this genetic structure to which the lineages correspond with their geographical distributions. For A. draco, $79 \%$ of the genetic variance was explained by PCA, axis 1 explained the genetic variation from W2d to W1d to E, and axis 2 explained the variation between W1d and W2d/E. For A. chevrieri, $54 \%$ of the genetic variance was explained by PCA, axis 1 gradually discriminated W2c to W1c, and E1c, and axis 2 mainly explained the genetic variation from E2c to E1c (Fig. 3 A3 and B3). In the IBD analyses of the two species, the genetic distance was highly correlated with the geographical distance when adjusted to the 'ring distance' (Mantel's test: A. draco , r=0.663; A. chevrieri , $\mathrm{r}=0.214$;) whereas the correlations with the straight distance were lower (Mantel's test: A. draco , $\mathrm{r}=0.169 ;$ A. chevrieri , $\mathrm{r}=0.033$;) (Fig 4). Furthermore, the cline analyses showed that for A. draco PC1 explained $55.6 \%$ of the genetic variance, and the mirrored exponential tails model was the best-fit model. The estimated cline center was located at $1308.02 \mathrm{~km}(1298.897-1317.146 \mathrm{~km}$, between sites HeS and WoL/BaX/GGS/LJS). The estimated cline width was $186.40 \mathrm{~km}(109.72-263.1 \mathrm{~km})$. For A. chevrieri , PC1 explained $24.31 \%$ of the genetic variance, and the left exponential tail model was the best fit model. The estimated cline center was located at $1394.2 \mathrm{~km}(1337-1451.47 \mathrm{~km}$, between sites GGS and $\mathrm{ZaT}$ ), and the estimated cline width was $351 \mathrm{~km}(65.7-638 \mathrm{~km})$ (Fig. 5).

In the diyABC analyses, we first ruled out two possible scenarios of the dispersal routes for the ancestral populations based on a comparative analysis of posterior probabilities using a logistic regression: For $A$. draco, (a) W2d is the ancestral population, and both W1d and E1d were independently derived from W2d (scenario 2); (b) E1d is the ancestral population, and W2d was derived from W1d, which itself was derived from E1d(scenario 3). For A. chevrieri, (a) W2c is the ancestral population, and E1c was derived from W1c which itself was derived from W2c (scenario 2); (b) E1c is the ancestral population, and W2c was derived from W1c, which itself was derived from E2c (scenario 3). In turn, scenarios were established in which W1c is the ancestral population of $A$. draco and both W2c and E1c were derived independently from W1c (scenario 1 ), and in which W1c is the ancestral population of A. chevrieri, and both W2c and E1c were derived from W1c (scenario 1). Next, based on the most appropriate scenario among W1, W2 and E1 in the first step, we added the fourth population E2 to deduce the entire evolutionary history of the two species around the Sichuan Basin. The model checking analyses support scenario 1 as the appropriate scenario for A. draco ; namely, E2d was derived from E1d, which itself was derived from W1d, and then W2d was derived from W1d. Scenario 3 is the appropriate scenario for A. chevrieri, namely, W2c and E1c were derived from W1c, and then there was an admixture event between the two populations, generating an admixed population, E2c (Fig. 3 A4 and B4; Fig. S1).

In the IMap2 analyses, asymmetric gene flow was observed between parapatric subclades. For $A$. draco, the gene flow among different parapatric subclades was largely different $(2 N M=102.5-0.135)$; the strong one was detected from E2d to E1d, and the weak one was detected from W2d to E2d. For A. chevrieri, the gene flow from E1c to E2c was strong $(2 N M=16.7678)$, and it was relatively weak between population W2c and E2c $(2 \mathrm{NM}=0.0122-0.0014)$ (Table S5)

In the RDA of the two species, both genetic structures were mainly interpreted according to the combined environmental and spatial variables model. That for A.dracoexplained $36.5 \%$ of the variance, and that for A. chevrieri explained $38.4 \%$, whereas environmental variables without spatial influence or spatial variables without environmental influence showed low explanatory power (Table 1). For A. draco, the main influence on the West group was PET, parts of both the West and Northwest groups had a high positive correlation with elevation and precipitation seasonality; the North group had a high positive correlation with spatial variables, temperature seasonality, and precipitation of the warmest quarter; and part of the three groups 
had a high correlation with NPP. For A. chevrieri, the West group had a positive correlation with NPP, PET and elevation; the Northwest group was affected by the mean diurnal range; and the North group had a positive correlation with spatial temperature and precipitation of the driest month (Fig. 6; Fig. S2).

Species distribution predicted by modeling

The predicted potential and suitable distributional range under the current and LIG/LGM climate conditions are shown in Fig. 7. All models had high AUC test values $(>0.95)$, indicating a good performance of all predictions. The results showed that the majority of the Sichuan Basin was always uninhabitable for A. draco and A. chevrieri under current and LIG/LGM climatic conditions, and the potential suitable distribution areas generally occurred along the mountain ranges surrounding the basin. Compared with the current habitat, the suitable habitat of the two species likely slightly expanded during the LIG and contracted during the LGM, especially in the northern and western periphery of the basin.

\section{Discussion}

Our study showed that bothApodemus draco and A. chevrieridisplay a ring diversification pattern around the Sichuan Basin, mediated by isolation by isolation, based on the solid evidence of comprehensive phylogeographic analyses. We inferred that this evolutionary process was driven by environmental heterogeneity and spatial variation, which were triggered by a combination of tectonic events of the QTP and climatic oscillations during the Quaternary. The process was also impacted by species traits. By identifying the consistency of the timeline between the diversification periods of the two species, the tectonic events and climatic oscillations, and the relationship between genetic structure and the environmental/spatial factors. Our results provided supportive evidence for the hypothesis that speciation is a continuous process in nature and supported that the ring-like manner of diversification is a general feature of evolution when the candidate taxa are 'fit' to the barriers, and there are favorable conditions conducive to divergence.

Ring distribution and diversification rather than ring species

The comprehensive phylogeographic analyses indicated both $A$. dracoand A. chevrieri have a ring pattern of genetic differentiation around the geographic barrier (Sichuan Basin), which is similar to the 'broken' ring species or ring diversification pattern described for theAlophoixus bulbuls (Fuchs et al., 2015) and the eastern tree frog (H.orientalis ) (Dufresnes et al., 2016).

First, both $A$. draco and A. chevrieri exhibited contemporary and historical patterns of a ring-shaped distribution. As montane species, the two species shifted upslope and downslope of the mountains to mitigate the impact of climate oscillations (Fjeldsa, Bowie, \& Rahbek, 2012; He, Hu, Chen, Li, \& Jiang, 2016; Hewitt, 1996; Wu, Dubay, Colwell, Ran, \& Lei, 2017), but did not retreat into the Sichuan Basin even during the LGM (Fig. 7), whereas the odorous frog (Odorrana margaretae) (another species distributed around the Sichuan Basin) retreated into the refugia (Sichuan Basin) during the LGM and returned to the mountains as the climate became warmer due to its relatively lower elevational distribution (390-1650 $\mathrm{m}$ ) versus the twoApodemus species (Fei, Hu, Ye, \& Huang, 2009) (Qiao et al., 2018). This pattern was verified by the projections of the Pleistocene and current distribution through the SDM analyses, and the models indicated the long-term existence of inhospitable habitat in the center of the ring to prevent gene flow through the basin. Moreover, the genetic distances were obviously more highly correlated with the ring distance than with the straight distance across the basin (Fig. 4), indirectly confirming the persistence of the barrier effect. The persistence of the barrier effect and relatively stable ring-shaped distribution is fundamental to the discussion of ring species/ring diversification (Dufresnes et al., 2016; Irwin, Bensch, Irwin, \& Price, 2005).

Second, a general trend of genetic divergence was detected in the genetic structure analyses for $A$. dracoand $A$. chevrieri, namely, a split into western and eastern lineages based on the nuclear data (Fig. 3). Their geographic distribution was highly congruent with that of the four clades clustered based on mtDNA data (Fig. 1), namely, the western lineages corresponding to mtDNA clades D1 and D4 of $A$. draco , and C1 and $\mathrm{C} 2$ of $A$. chevrieri and the eastern lineages corresponding to clades D2 and D3 of A. draco, and 
$\mathrm{C} 3$ and $\mathrm{C} 4$ of $A$. chevrieri . Other taxa distributed in this region also exhibit a western and eastern divergence, one example is the giant panda (Ailuropoda melanoleuca), which is morphologically split into the Sichuan cluster (western) and Qinling cluster (eastern) (Wan, Wu, \& Fang, 2005). In particular, the populations of riparian shrub (Sophora davidii) (Fan et al., 2013) and the odorous frog (O. margaretae) (Qiao et al., 2018)around the Sichuan Basin not only present a west-east genetic divergence pattern but also had remarkably similar split times (approximately 1.1-1.7 Mya) with the two Apodemusspecies. These similarities point to a common underlying driver of genetic divergence. In the IBD analyses, the two species showed gradual genetic mutation along with geographical Euclidian distance and present continuous levels of population divergence, this genetic pattern has been observed in many ring species (Alcaide et al., 2014; Irwin et al., 2005; Qiao et al., 2018).

Third, the dispersal routes support the prediction that the western lineages of $A$. draco and A. chevrieridispersed clockwise from west to northwest, while the eastern lineage dispersed anticlockwise from west to north, then, the two lineages met approximately in the northwest region (Fig. 3). The cline analysis further verified that both the hybrid zones of $A$. draco and $A$. chevr ieriwere located in the northwest (the hybrid zone of $A$. draco was more northwestern than $A$. chevrieri) and corresponded to their geographic group W2 which represented the dispersal end of western lineages (Fig. 5). Intriguingly, both groups W2 of the two species belonged to the western lineage based on the nuclear DNA but had mitochondrial DNA from the eastern clades D4 and C2, suggesting a history of sex-asymmetric hybridization and selection for a particular mitochondrial type in the mixed population (Irwin, 2016). The asymmetric gene flow observed between group W2 and E2 were in support of gene exchange between the terminals of the western and eastern lineages, and the reduced level gene flow at the terminal of the rings support the existence of partial reproductive isolation (Table S5). The STRUCTURE analyses $(K=2)$ showed that some individuals in the W2 population of $A$. draco (site LJS, MiY, YaB and BaM) were almost entirely of the eastern lineage, and some exhibited a variety of intermediate values between the western and eastern lineages (Fig. 3A2). This suggests that these populations may have a large amount of assortative mating and may not be entirely blended, raising the possibility of some amount of partial reproductive isolation between the western and eastern lineages (Dufresnes et al., 2016; Hermansen et al., 2014). Full reproductive isolation is expected when the ring distributional ranges is extended and the divergence time is prolonged (Fuchs et al., 2015).

What triggered this ring-shaped diversification?

Geological movement was one of the original drivers of the genetic divergence of $A$. draco and A. chevrieri . In line with Ge et al. (2019), we showed that the divergence times of the two main clades (1.6 Mya for $A$. dracoand 1.1 Mya for A. chevrieri) and other subclades (all subdivisions occurred before $0.5 \mathrm{My}$ ) inferred based on the mitochondrial DNA, coincided with the uplift events of the QTP, which were known as the Qingzang Movement (3.6-1.7 Mya) and the Kun-Huang Movement (1.2-0.6 Mya) (Li \& Fang, 1999; Williams, Dunkerley, Deckker, Kershaw, \& Stokes, 1997). These tectonic events continually developed mountains and watercourses and supplied diversified topography for vicariance. In particular, the mountains adjacent to the northwestern Sichuan Basin show the most notable geomorphological changes as part of the QTP and have been directly affected; this region thus became the potential barrier belt for the clockwise dispersal of $A$. draco and A. chevrieri(Fig. 1; Fig. 3) and O. margaretae (Li et al., 1979; Qiao et al., 2018). Furthermore, the intense uplift of the QTP during the two events influenced the atmospheric circulation and promoted the formation of the modern monsoon circulation pattern. It subjected the HDMs (west) to the monsoons from both the Pacific and Indian oceans, and other mountains around the basin (east) to the Pacific monsoons, which contributed to the heterogeneity of the environment (Pan, Fang, Li, Shi, \& Cui, 1998; Williams et al., 1997).

Climatic oscillations during the Quaternary also played a major role in shaping the genetic structure of $A$. draco and A. chevrieri (Hewitt, 2000). Owing to the traits of the habitats of the two species and the unique geographical feature, the ring distributional patterns were maintained during the glacial period. However, the effect of climatic oscillations was not negligible even in species refugia, especially in terms of the genetic structure. The influence of climate oscillations was twofold: First, it placed abiotic pressures on species. 
When the evolution rate of the fundamental species' thermal niche was smaller than that of the temperature change, the species would shift its elevation range to track suitable temperature (Wu et al., 2017). Second, climatic oscillations would also affect biotic interactions (Colwell, 2008; Freeman, 2015; Lenoir, Gegout, Marquet, Ruffray, \& Brisse, 2008). As mentioned above, the small mammals (A. draco and A. chevrieri ) and frog (O. margaretae) (Qiao et al., 2018)around the Sichuan Basin seem to have a trend of westeast genetic divergence similar to that of a riparian shrub (Sophora davidii) (Fan et al., 2013), despite the boundaries being incompletely coincident. The major phylogeographic break for S. davidii was the 'Tanaka-Kaiyong Line' (TKL), which was a Pleistocene-originated environmental barrier to the dispersal of plants, whereby differing climate regimes were established on either side (Zhu \& Yan, 2002). The changing vegetation types further influenced the genetic divergence of animals.

How did

the ring diversification pattern around the Sichuan Basin take shape?

The geographical and climatic drivers caused the genetic divergence of $A$. draco and A. chevrieri by providing environmental heterogeneity and spatial variation. Environmental and spatial factors combined contributed to the formation of the population structure of $A$. draco and A. chevrieri(explaining $36.5 \%$ of the genetic variance for $A$. draco and $38.4 \%$ of the genetic variance for A. chevrieri ) (Table 1; Fig. 6) in the redundancy analyses, whereas environmental variables without spatial influence or spatial variables without environmental influence showed low explanatory power (Fig. S2). The consistent results of the two species verified the idea that the environmental and spatial factors were often spatially autocorrelated with one another (Myers et al., 2019). When focusing on the different contributions of variables to the three regional groups, we found that the genetic variation in the West and Northwest groups mainly presented a high correlation with elevation, and that of the North group showed a high correlation with spatial location and temperature. This difference can be explained by local adaptation and divergence. The West and Northwest groups were mainly distributed in the HDMs, the topography is complex, and the elevational difference in this region is large. A typical example is the Gongga Mountain, which spans an elevational range of more than $6000 \mathrm{~m}$. The North groups mainly occupy the YGP and Wu Mountains in the southeast and the Daba Mountains and Qinling Mountains in the north. These regions were characterized by a relatively simple topography and had lower elevations (mostly below 2,500 m) than the HDMs. A special karst landform is seen in the southeast region, and it is renowned for its high levels of endemism (Li, 1989; Zhu, 2007). The distinctive morphology and hydrology caused by the surface and subsurface rock dissolution drive the speciation via local adaptations (Guo et al., 2019; Kong, Condamine, Harris, Chen, \& Kang, 2017).

Species traits were also important factors in structuring the population genetic patterns (Zamudio, Bell, \& Mason, 2016). Lavinia et al. (2019) verified that the biological attributes of species made their evolutionary histories distinguishable by accessing the population divergence of two disjunctly codistributed birds (Ramphotrigon megacephalum andPipraeidea melanonota). The populations of different species surrounding the Sichuan Basin have similar genetic patterns, but the distinction cannot be ignored, even in closely genetic related and sympatric species, such as A. draco and A. chevrieri (Corbet \& Hill, 1992). Although they inhabit similar landscapes and experience similar types of environmental variation, the genetic structures of the two species are not the same, such as the details of split time and dispersal routes. The supposed explanation for this occurrence is the distinct traits of the two species, such as their home range size, degree of philopatry, and rate and distance of successful migration (Zamudio et al., 2016). Despite we have identified the correlations between the population genetic structures and spatial/environmental characteristics, examining the underlying relationship between genetic variation and species traits is difficult (Reid, Mladenoff, \& Peery, 2016).

When were species real 'ring species' around the Sichuan Basin?

When were species real 'ring species' around the Sichuan Basin? We may be able to find some clues in our current and previous studies, which generally showed that the formation of the rings depends on the interaction of many factors (e.g., split time, dispersal ability, and the size of the barrier). 
The organic integration of the speed of expansion $(v)$, the ring size $(L)$, and the rate of genetic isolation $(w)$ is vital to the formation of 'ring species'. A study based on simulations inferred that ring species will form if the period of expansion $(L w / v)$ is approximately 1 (Martins, Aguiar, \& Bar-Yam, 2013). Relatively mobile species require larger barriers (e.g., greenish warblers around the Tibetan Plateau and Alophoixus bulbuls around the Thailand lowlands) to form the rings. For a small geographic barrier, only species with limited dispersal ability (e.g.H.orientalis around the Black Sea, and A. draco and A. chevrieriaround the Sichuan Basin) are candidates for displaying rings. Even if the above conditions ( $L w / v[?] 1)$ are met, the formation of ring species requires driving forces, such as the geographic heterogeneity caused by the uplift of the QTP and the environmental heterogeneity caused by the modern monsoon circulation pattern. Moreover, climatic oscillations during the Quaternary also supplied the opportunity for the divergence of these rings (Dufresnes et al., 2016; Fuchs et al., 2015; Qiao et al., 2018). The influence of these genetic divergence drivers is also closely related to the split time of these species. Supposing that the time of the split of the two species from their sister species was much earlier or later than that of the Qingzang Movement and the Kun-Huang Movement, the divergence of the western and eastern lineages may not have occurred as a result of the absence of an impact of tectonic movement and subsequent associated environmental change. When the conditions mentioned above are met, 'ring species' may occur.

\section{Acknowledgements}

We express our appreciation to the editors and anonymous reviewers for their careful review and constructive comments and suggestions. We sincerely thank Alexei V. Abramov, Yuanbao Du, Brawin Kumar, Kangli Zhu and Feihong Li for their help with the fieldwork. We thank Fangyuan Yang, Kai He, Dezhi Zhang, Jinzhong Fu and Jie Gao for their suggestions of the analyses. This research was supported by the National Natural Science Foundation of China (No.31801964 and 31900325) and the Key Laboratory of Zoological Systematics and Evolution of the Chinese Academy of Sciences (No. Y229YX5105).

\section{Author Contributions}

Q.Y., Y.W., L.X. and J.C. designed and contributed to the original concept of the studies. D.G., J.C., Z.W., J.S., S.L. and L.L. collected most of the specimens and related data, A.F. and Y.W. conducted the cline analysis and RDA, J. C. and Y.W. conducted the SDM analysis, Y.W. completed most other data analysis and finalized the manuscript. All authors participated in project design, and read and approved the final manuscript.

\section{Data Accessibility}

Microsatellite DNA data have been uploaded as online supporting information (Appendix S2). All sequences have been deposited in GenBank (accession numbers MT197600-MT198019 for Cytb of Apodemus draco , MT198023-MT198218 for COI of A. draco , MT187460-MT187584 forCytb of A. chevrieri, MT219316MT219445 for COI of $A$. chevrieri) (Appendix S1).

\section{References}

Alcaide, M., Scordato, E. S., Price, T. D., \& Irwin, D. E. (2014). Genomic divergence in a ring species complex. Nature, 511 (7507), 83-85.

Bennett, K. D., \& Provan, J. (2008). What do we mean by 'refugia'? Quaternary Science Reviews, 27 (27-28), 2449-2455.

Chen, Z., He, K., Cheng, F., Khanal, L., \& Jiang, X. (2017). Patterns and underlying mechanisms of non-volant small mammal richness along two contrasting mountain slopes in southwestern China. Scientific Reports, 7 (1), 13277.

Colwell, R. K. (2008). RangeModel: tools for exploring and assessing geometric constraints on species richness (the mid-domain effect) along transects. Ecography, 31 (1), 4-7. 
Corbet, G. B., \& Hill, J. E. (1992). The mammals of the Indomalayan Region: a systematic review . London: Oxford University Press.

Cornuet, J. M., Pudlo, P., Veyssier, J., Dehne-Garcia, A., Gautier, M., Leblois, R., . . . Estoup, A. (2014). DIYABC v2.0: a software to make approximate Bayesian computation inferences about population history using single nucleotide polymorphism, DNA sequence and microsatellite data. Bioinformatics, 30 (8), 1187-1189.

Derryberry, E. P., Derryberry, G. E., Maley, J. M., \& Brumfield, R. T. (2014). hzar: hybrid zone analysis using an R software package.Molecular Ecology Resources, 14 (3), 652-663.

Devitt, T. J., Baird, S. J. E., \& Moritz, C. (2011). Asymmetric reproductive isolation between terminal forms of the salamander ring species Ensatina eschscholtzii revealed by fine-scale genetic analysis of a hybrid zone. BMC Evolutionary Biology, 11 (1), 245-257.

Drummond, A. J., \& Rambaut, A. (2007). BEAST: Bayesian evolutionary analysis by sampling trees. BMC Evol Biol, 7 (7), 214.

Dufresnes, C., Litvinchuk, S. N., Leuenberger, J., Ghali, K., Zinenko, O., Stock, M., \& Perrin, N. (2016). Evolutionary melting pots: a biodiversity hotspot shaped by ring diversifications around the Black Sea in the Eastern tree frog (Hyla orientalis). Mol Ecol, 25 (17), 4285-4300.

Earl, D. A., \& Vonholdt, B. M. (2012). STRUCTURE HARVESTER: a website and program for visualizing STRUCTURE output and implementing the Evanno method. Conservation Genetics Resources, 4 (2), 359-361.

Elith, J. (2000). Quantitative Methods for Modeling Species Habitat: Comparative Performance and an Application to Australian Plants. 39-58.

Evanno, G., Regnaut, S., \& Goudet, J. (2005). Detecting the number of clusters of individuals using the software structure: a simulation study. Molecular Ecology, 14 (8), 2611-2620.

Excoffier, L., \& Lischer, H. E. (2010). Arlequin suite ver 3.5: a new series of programs to perform population genetics analyses under Linux and Windows. Molecular Ecology Resources, 10 (3), 564-567.

Fan, D., Yue, J., Nie, Z., Li, Z., Comes, H. P., \& Sun, H. (2013). Phylogeography of Sophora davidii (Leguminosae) across the 'Tanaka-Kaiyong Line', an important phytogeographic boundary in Southwest China. Molecular Ecology, 22 (16), 4270-4288.

Fan, Z., Liu, S., Liu, Y., Liao, L., Zhang, X., \& Yue, B. (2012). Phylogeography of the South China field mouse (Apodemus draco) on the southeastern Tibetan Plateau reveals high genetic diversity and glacial refugia. PloS one, 7 (5), e38184.

Fei, L., Hu, S., Ye, C., \& Huang, Y. (2009). Fauna Sinica, Amphibia (Vol. 3). Beijing, China: Science Press.

Fick, S. E., \& Hijmans, R. J. (2017). WorldClim 2: new 1-km spatial resolution climate surfaces for global land areas. International Journal of Climatology, 37 (12), 4302-4315.

Fjeldsa, J., Bowie, R. C. K., \& Rahbek, C. (2012). The Role of Mountain Ranges in the Diversification of Birds. Annual Review of Ecology, Evolution, and Systematics, 43 (1), 249-265.

Forester, B. R., Lasky, J. R., Wagner, H. H., \& Urban, D. L. (2018). Comparing methods for detecting multilocus adaptation with multivariate genotype-environment associations. Molecular Ecology, 27 (9), $2215-2233$.

Foster, S. A., Scott, R. J., \& Cresko, W. A. (1998). Nested biological variation and speciation. Philosophical Transactions of the Royal Society B: Biological Sciences, 353 (1366), 207-218.

Freeman, B. G. (2015). Thermal tolerances to cold do not predict upper elevational limits in New Guinean montane birds. Diversity and Distributions, 22 (3), 309-317. 
Fuchs, J., Ericson, P. G. P., Bonillo, C., Couloux, A., \& Pasquet, E. (2015). The complex phylogeography of the Indo-Malayan Alophoixus bulbuls with the description of a putative new ring species complex.Molecular Ecology, 24 (21), 5460-5474.

Ge, D. Y., Feijo, A., Cheng, J. L., Lu, L., Liu, R. R., Abramov, A. V., . . . Yang, Q. S. (2019). Evolutionary history of field mice (Murinae: Apodemus), with emphasis on morphological variation among species in China and description of a new species. Zoological Journal of the Linnean Society, 187 (2), 518-534.

Glor, R. E., \& Warren, D. L. (2011). Testing ecological explanations for biogeographic boindaries. Evolution, $65(3), 673-683$.

Gu, X. D., Liu, S. Y., Wang, Y. Z., \& Wu, H. (2009). Development and characterization of eleven polymorphic microsatellite loci from South China field mouse (Apodemus draco). Conservation genetics, 10 (6), 1961-1963.

Guindon, S., \& Gascuel, O. (2003). A simple, fast, and accurate algorithm to estimate large phylogenies by maximum likelihood.Systematic Biology, 52 (5), 696-704.

Guo, Y. L., Chen, Y. H., Mallik, A. Z., Wang, B., Li, D. X., Xiang, W. S., \& Li, X. K. (2019). Predominance of abiotic drivers in the relationship between species diversity and litterfall production in a tropical karst seasonal rainforest. Forest Ecology and Management, 449, 117452.

He, K., Gutierrez, E. E., Heming, N. M., Koepfli, K. P., Wan, T., He, S. W., . . . Jiang, X. L. (2019). Cryptic phylogeographic history sheds light on the generation of species diversity in sky-island mountains. Journal of Biogeography, 46 (10), 2232-2247.

He, K., Hu, N. Q., Chen, X., Li, J. T., \& Jiang, X. L. (2016). Interglacial refugia preserved high genetic diversity of the Chinese mole shrew in the mountains of southwest China. Heredity, 116 (1), 23-32.

Hermansen, J. S., Haas, F., Trier, C. N., Bailey, R. I., Nederbragt, A. J., Marzal, A., \& Saetre, G. P. (2014). Hybrid speciation through sorting of parental incompatibilities in Italian sparrows. Molecular Ecology, 23 (23), 5831-5842.

Hewitt, G. (2000). The genetic legacy of the Quaternary ice ages.Nature, 405 (6789), 907-913.

Hewitt, G. M. (1996). Some genetic consequences of ice ages, and their role in divergence and speciation . Biological Journal of the Linnean Society, 58 (3), 247-276.

Irwin, D. E. (2016). A tree of tree frogs around the Black Sea.Molecular Ecology, 25 (17), 4093-4096.

Irwin, D. E., Bensch, S., Irwin, J. H., \& Price, T. D. (2005). Speciation by Distance in a Ring Species. Science, 307 (5708), 414-416.

Irwin, D. E., Bensch, S., \& Price, T. D. (2001). Speciation in a ring.Nature, 409 (6818), 333-337.

Irwin, D. E., Irwin, J. H., \& Price, T. D. (2001). Ring species as bridges between microevolution and speciation. Genetica, 112 (1), 223-243.

Irwin, D. M., Kocher, T. D., \& Wilson, A. C. (1991). Evolution of the cytochrome b gene of mammals. $J$ Mol Evol, 32 (2), 128-144.

Johnson, N. M., Stix, J., Tauxe, L., Cerveny, P. F., \& Tahirkheli, R. A. K. (1985). Paleomagnetic Chronology, Fluvial Processes, and Tectonic Implications of the Siwalik Deposits near Chinji Village, Pakistan.Journal of Geology, 93 (1), 27-40.

Jombart, T., Devillard, S., \& Balloux, F. (2010). Discriminant analysis of principal components: a new method for the analysis of genetically structured populations. BMC Genetics, 11 (1), 94.

Joseph, L., Dolman, G., Donnellan, S., Saint, K. M., Berg, M. L., \& Bennett, A. T. D. (2008). Where and when does a ring start and end? Testing the ring-species hypothesis in a species complex of Australian 
parrots. Proceedings of the Royal Society B Biological Sciences, 275 (1650), 2431.

Kierepka, E. M., \& Latch, E. K. (2015). Performance of partial statistics in individual-based landscape genetics. Mol Ecol Resour, 15 (3), 512-525.

Kimura, Y., Flynn, L. J., \& Jacobs, L. L. (2017). Early Late Miocene Murine Rodents from the Upper Part of the Nagri Formation, Siwalik Group, Pakistan, with a new fossil calibration point for the Tribe Apodemurini (Apodemus/Tokudaia) Fossil Imprint, 73 , 197-212.

Kong, H. H., Condamine, F. L., Harris, A. J., Chen, J. L., Pan, B., Moller, M., . . . Kang, M. (2017). Both temperature fluctuations and East Asian monsoons have driven plant diversification in the karst ecosystems from southern China. Molecular Ecology, 26 (22), 6414-6429.

Kuchta, S. R., Parks, D. S., \& Wake, D. B. (2009). Pronounced phylogeographic structure on a small spatial scale: Geomorphological evolution and lineage history in the salamander ring species Ensatina eschscholtzii in central coastal California. Molecular phylogenetics and evolution, 50 , 240-255.

Lavinia, P. D., Barreira, A. S., Campagna, L., Tubaro, P. L., \& Lijtmaer, D. A. (2019). Contrasting evolutionary histories in Neotropical birds: Divergence across an environmental barrier in South America. Mol Ecol, 28 (7), 1730-1747.

Lenoir, J., Gegout, J. C., Marquet, P. A., Ruffray, P. d., \& Brisse, H. (2008). A Significant Upward Shift in Plant Species Optimum Elevation During the 20th Century. Science, 320 (5884), 1768-1771.

Li, B. (1989). Geomorphologic regionalization of the Hengduan mountainous region. Journal of Mountain Research, 7 (1), 14-20.

Li, J., Zheng, X., Cai, Y., Zhang, X., Yang, M., Yue, B., \& Li, J. (2015). DNA barcoding of Murinae (Rodentia: Muridae) and Arvicolinae (Rodentia: Cricetidae) distributed in China. Molecular Ecology Resources, 15 (1), 153-167.

Li, J. J., \& Fang, X. M. (1999). Uplift of the Tibetan Plateau and environmental changes. Chinese Science Bulletin, 44 (23), 2117-2124.

Li, J. J., Wen, S. X., Zhang, Q. S., Wang, F. B., Zhang, B. X., \& Li, B. Y. (1979). A discussion on the period,amplitude and type of the uplift of the Qinghai-Xizang Plateau. Scientia Sinica, 22 , 1314-1328.

Liebers, D., Knijff, P. d., \& Helbig, A. J. (2004). The herring gull complex is not a ring species. Proceedings Biological Sciences, 271 (1542), 893-901.

Liu, Q., Chen, P., He, K., Kilpatrick, C. W., Liu, S. Y., Yu, F. H., \& Jiang, X. L. (2012). Phylogeographic study of Apodemus ilex (Rodentia: Muridae) in Southwest China. PloS one, 7 (2), e31453.

Liu, S. G., Deng, B., Li, Z. W., \& Sun, W. (2012). Architecture of basin-mountain systems and their influences on gas distribution: A case study from the Sichuan basin, South China. Journal of Asian Earth Sciences, 47 , 204-215.

Liu, S. Y., He, K., Chen, S. D., Jin, W., Murphy, R. W., Tang, M. K., . . . Li, F. J. (2018). How many species of Apodemus and Rattus occur in China? A survey based on mitochondrial cyt b and morphological analyses.39 (5), 309-320.

Liu, X., Wei, F., Li, M., \& Feng, Z. (2002). A review of the phylogenetic study on the genus Apodemus of China. Acta Theriologica Sinica, 22 (1), 46-52.

Liu, X., Wei, F., Li, M., Jiang, X., Feng, Z., \& Hu, J. (2004). Molecular phylogeny and taxonomy of wood mice (genus Apodemus Kaup, 1829) based on complete mtDNA cytochrome b sequences, with emphasis on Chinese species. Molecular phylogenetics and evolution, 33 (1), 1-15. 
Lv, X., Cheng, J., Meng, Y., Chang, Y., Xia, L., Wen, Z., . . . Yang, Q. (2018). Disjunct distribution and distinct intraspecific diversification of Eothenomys melanogaster in South China. BMC Evolutionary Biology, 18 (1), 50-64.

Martins, A. B., de Aguiar, M. A., \& Bar-Yam, Y. (2013). Evolution and stability of ring species. Proceedings of the National Academy of Sciences of the United States of America, 110 (13), 5080-5084.

Mayr, E. (1942). Systematics and the Origin of Species . New York City, New York: Columbia University Press.

Monahan, W. B., Pereira, R. J., \& Wake, D. B. (2012). Ring distributions leading to species formation: a global topographic analysis of geographic barriers associated with ring species. BMC Biology, 10 , 20-20.

Myers, E. A., Xue, A. T., Gehara, M., Cox, C. L., Davis Rabosky, A. R., Lemos-Espinal, J., . . Burbrink, F. T. (2019). Environmental heterogeneity and not vicariant biogeographic barriers generate community-wide population structure in desert-adapted snakes. Molecular Ecology, 28 (20), 4535-4548.

Myers, N., Mittermeier, R. A., Mittermeier, C. G., da Fonseca, G. A., \& Kent, J. (2000). Biodiversity hotspots for conservation priorities. Nature, 403 (6772), 853-858.

Pan, B. T., Fang, X., Li, J. J., Shi, Y. F., \& Cui, Z. J. (1998). Uplift and environmental changes of the Qinghai-Xizang (Tibetan) Plateau during the Late Cenozoic Period. Uplift and Environmental Changes of Qinghai-Xizang (Tibetan) Plateau in the Late Cenozoic, 373-414.

Peakall, R., \& Smouse, P. E. (2012a). GenAlEx 6.5: genetic analysis in Excel. Population genetic software for teaching and research-an update. Bioinformatics, 28 (19), 2537-2539.

Peakall, R., \& Smouse, P. E. (2012b). GenAlEx 6.5: genetic analysis in Excel. Population genetic software for teaching and research-an update.Bioinformatics, 28 (19), 2537-2539.

Pereira, R. J., \& Wake, D. B. (2015). Ring species as demonstrations of the continuum of species formation. Molecular Ecology, 24 (21), 5312-5314.

Petit, R. J., Itziar, A., Jacques-Louis, D. B., Christiane, B., Simon, B., Rachid, C., . . . Martin, L. (2003). Glacial refugia: hotspots but not melting pots of genetic diversity. Science, 300 (5625), 1563-1565.

Phillips, S. J., Anderson, R. P., \& Schapire, R. E. (2006). Maximum entropy modeling of species geographic distributions. Ecological Modelling, 190 (3-4), 231-259.

Posada, D., \& Crandall, K. A. (2001). Intraspecific gene genealogies: trees grafting into networks. Trends in Ecology 83 Evolution, 16 (1), 37-45.

Pritchard, J. K., Stephens, M., \& Donnelly, P. (2000). Inference of population structure using multilocus genotype data. Genetics, 155 (2), 945-959.

Provan, J., \& Bennett, K. D. (2008). Phylogeographic insights into cryptic glacial refugia. Trends in Ecology ES Evolution, 23 (10), 564-571.

Qiao, L., Wen, G., Qi, Y., Lu, B., Hu, J., Song, Z., \& Fu, J. (2018). Evolutionary melting pots and reproductive isolation: A ring-shaped diversification of an odorous frog (Odorrana margaratea) around the Sichuan Basin. Molecular Ecology, 27 (23), 4888-4900.

Rambaut, A., Drummond, A. J., Xie, D., Baele, G., \& Suchard, M. A. (2018). Posterior Summarization in Bayesian Phylogenetics Using Tracer 1.7. Systematic Biology, 67 (5), 901-904.

Raymond, M., \& Rousset, F. (1995). Genepop (Version-1.2) - Population-Genetics Software for Exact Tests and Ecumenicism. Journal of Heredity, 86 (3), 248-249.

Reid, B. N., Mladenoff, D. J., \& Peery, M. Z. (2016). Genetic effects of landscape, habitat preference, and demography on three co-occurring turtle species. Molecular Ecology, 26 (3), 781-791. 
Rice, W. R., \& Hostert, E. E. (1993). Laboratory Experiments on Speciation: What Have We Learned in 40 Years? Evolution, 47 (6), 1637-1653.

Ronquist, F., Teslenko, M., van der Mark, P., Ayres, D. L., Darling, A., Hohna, S., . . . Huelsenbeck, J. P. (2012). MrBayes 3.2: Efficient Bayesian Phylogenetic Inference and Model Choice Across a Large Model Space. Systematic Biology, 61 (3), 539-542.

Rousset, F. (2008). GENEPOP (version 4.0) GENEPOP'007: a complete re-implementation of the genepop software for Windows and Linux.Molecular Ecology Resources, 8 (1), 103-106.

Sakka, H., Quere, J. P., Kartavtseva, I., Pavlenko, M., Chelomina, G., Atopkin, D., . . . Michaux, J. (2010). Comparative phylogeography of four Apodemus species (Mammalia: Rodentia) in the Asian Far East: evidence of Quaternary climatic changes in their genetic structure.Biological Journal of the Linnean Society, 100 (4), 797-821.

Suzuki, H., Filippucci, M. G., Chelomina, G. N., Sato, J. J., Serizawa, K., \& Nevo, E. (2008). A Biogeographic View of Apodemus in Asia and Europe Inferred From Nuclear and Mitochondrial Gene Sequences.Biochemical Genetics, 46 (5-6), 329.

Suzuki, H., Sato, J. J., Tsuchiya, K., Luo, J., Zhang, Y. P., Wang, Y. X., \& Jiang, X. L. (2015). Molecular phylogeny of wood mice (Apodemus, Muridae) in East Asia. Biological Journal of the Linnean Society, 80 (3), 469-481.

Swets, J. (1988). Measuring the accuracy of diagnostic systems.Science, 240 (4857), 1285-1293.

Synes, N. W., \& Osborne, P. E. (2011). Choice of predictor variables as a source of uncertainty in continentalscale species distribution modelling under climate change. Global Ecology $\& 3$ Biogeography, 20 (6), 904-914.

Van Oosterhout, C., Hutchinson, W. F., Wills, D. P. M., \& Shipley, P. (2004). micro-checker: software for identifying and correcting genotyping errors in microsatellite data. Molecular Ecology Notes, 4 (3), 535-538.

Wan, Q. H., Wu, H., \& Fang, S. G. (2005). A New Subspecies of Giant Panda (Ailuropoda melanoleuca) from Shaanxi, China. Journal of Mammalogy, 86 (2), 397-402.

Wan, T., He, K., Jin, W., Liu, S., \& Jiang, X. (2018). Climate niche conservatism and complex topography illuminate the cryptic diversification of Asian shrewike moles. Journal of Biogeography (2), 1-15.

Warren, D. L., Glor, R. E., \& Turelli, M. ENMTools: a toolbox for comparative studies of environmental niche models. Ecography, 33 (3), 607-611.

Williams, M. A. J., Dunkerley, D. L., Deckker, P. D., Kershaw, A. P., \& Stokes, T. J. (1997). Quaernary Environments (L. Dongsheng, Trans.). Beijing: Science Press.

Wu, H., Zhan, X. J., Yan, L., Liu, S. Y., Zhang, Z. J., Li, M., \& Wei, F. W. (2009). Isolation and characterization of 14 microsatellite loci for the Chevrier's field mouse (Apodemus chevrieri). Molecular Ecology Resources, 9 (3), 848-850.

Wu, Y., Dubay, S. G., Colwell, R. K., Ran, J., \& Lei, F. (2017). Mobile hotspots and refugia of avian diversity in the mountains of south-west China under past and contemporary global climate change. Journal of Biogeography, 44 (3).

Wu, Y., Xia, L., Zhang, Q., \& Yang, Q. (2010). Habitat fragmentation affects genetic diversity and differentiation of the Yarkand hare.Conservation genetics, 11 (1), 183-194.

Yue, H., Fan, Z., Liu, S., Liu, Y., Song, Z., \& Zhang, X. (2012). A mitogenome of the Chevrier's field mouse (Apodemus chevrieri) and genetic variations inferred from the cytochrome b gene. DNA and Cell Biology, 31 (4), 460-469. 
Zamudio, K. R., Bell, R. C., \& Mason, N. A. (2016). Phenotypes in phylogeography: Species' traits, environmental variation, and vertebrate diversification. Proceedings of the Natlonal Academy of Sciences of the United States of America, 113 (29), 8041-8048.

Zhang, M. C., Zhang, Z. J., Mu, H. Q., Huang, X. F., \& Hu, J. C. (2008). Geographical differentiation in skull morphology of isolated Apodemus chevrieri populations in western Sichuan Mountains. Acta Theriologica Sinica, 28 (4), 395-401.

Zhu, H. (2007). The karst ecoystem of southern China and its biodiversity. Tropical Forestry, 35 , 44-47.

Zhu, H., \& Yan, L. C. (2002). A discussion on biogeographical lines of the tropical-subtropical Yunnan. Chinese Geographical Science, 123 (1), 90-96.

Zhu, W. L., Jiang, W. X., Zhang, H., \& Wang, Z. K. (2014). Geometric morphometrics investigation of the skulls in Apodemus chevrieri from Hengduan mountain region. Journal of Biology, 31 (1), 37-40.

Tables and Figures

Fig. 1Map of southwest China with the distribution of mitochondrial clades for A. draco (A) and A. chevrieri (B). Pie sizes are proportional to sample sizes. Colors correspond to the phylogenetic tree in the top-right frame.

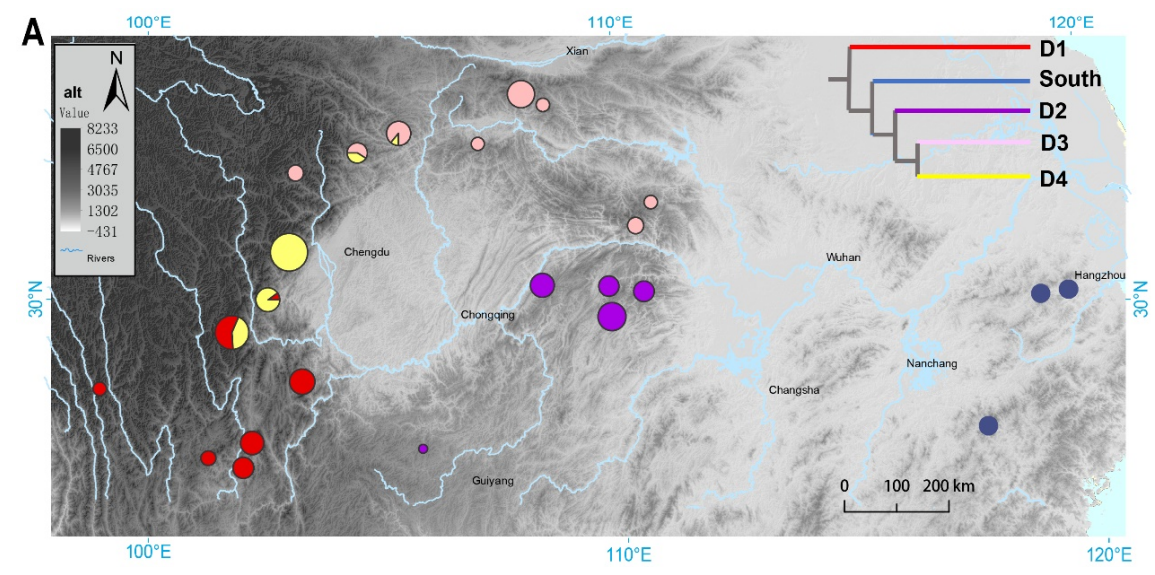




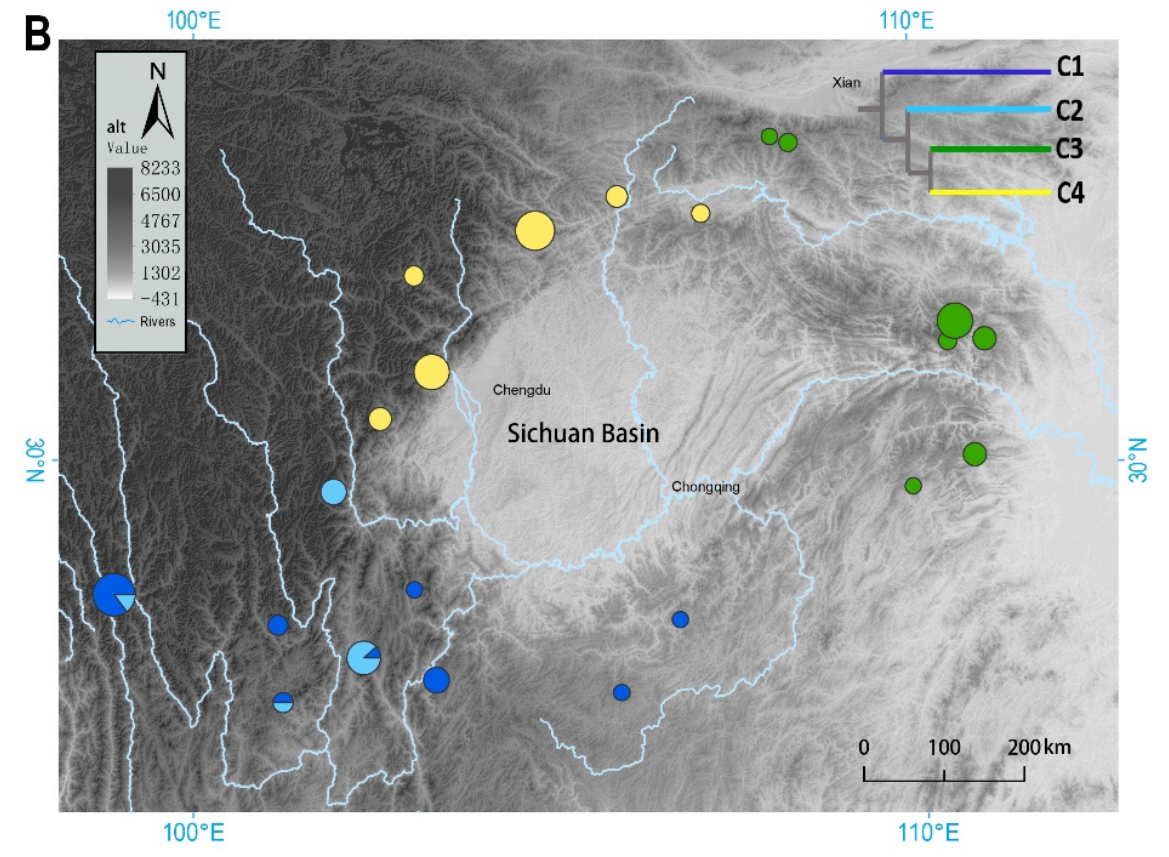

Fig. 2 Mitochondrial phylogenetic tree and divergence time of A. draco (A) and A. chevrieri (B), based on Cytband COI sequence variation. Posterior probabilities (Bayesian) are provided for major nodes. Arrows indicate divergence time estimates and their $95 \%$ posterior distributions. 


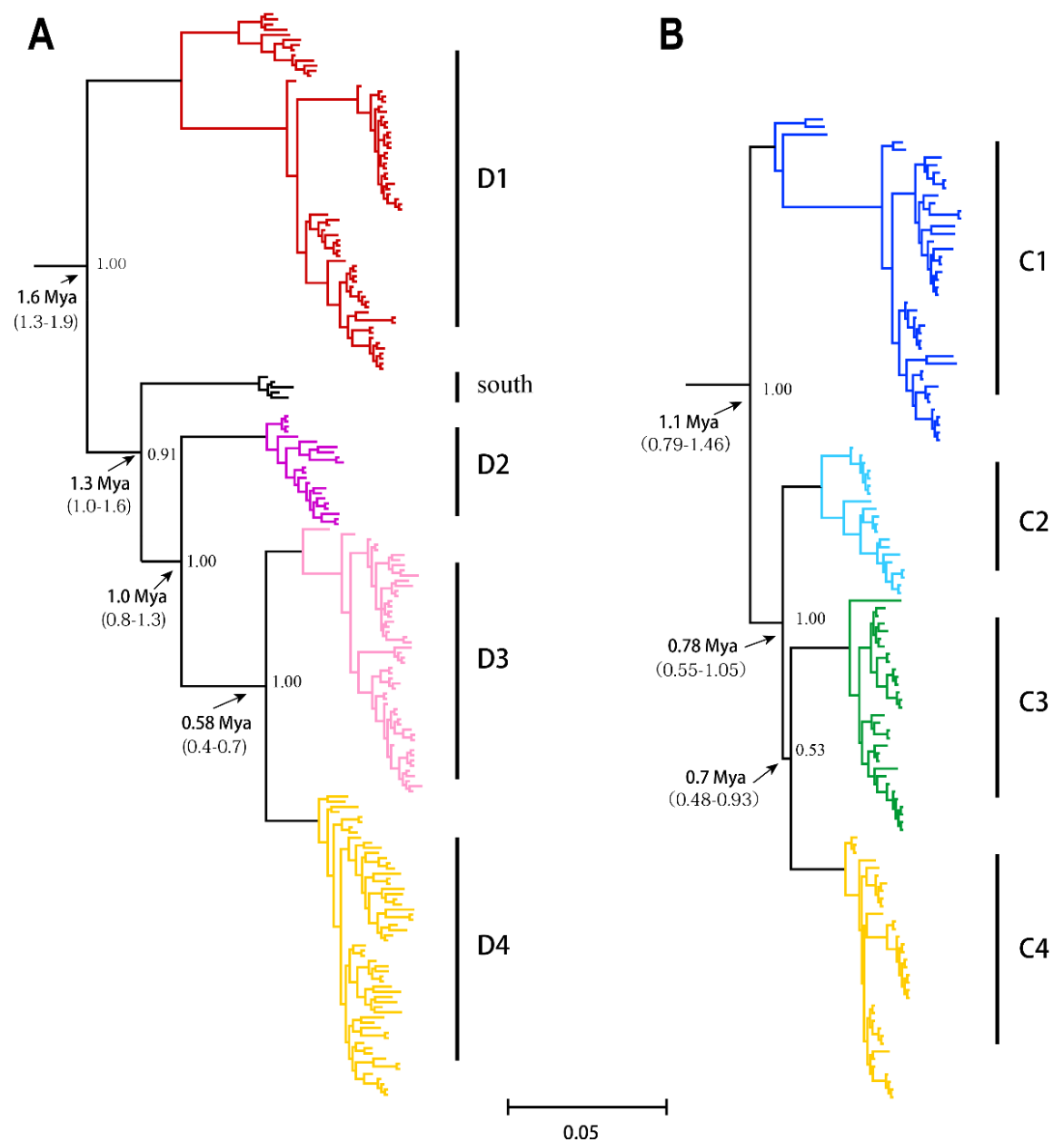

Fig. 3 Nuclear genetic differentiation in A. $\operatorname{draco}(\mathrm{A})$ and $A$. chevrieri (B) based on microsatellites data. (A1/B1) Average STRUCTURE ancestry coefficients of each population with $K=2$ (the best fit of our data). The colors are concordant with those of the corresponding clades in STRUCTURE. The dashed line shows the geographical groups when $K=4$. (A2/B2) Bayesian clustering of microsatellite genotypes using STRUCTURE. Analyses are shown for $K=2$ and $K=3$. (A3/B3) First axes of discriminant analysis of principal components (DAPC) of populations' allele frequencies. (A3/B3) The most appropriate scenarios of the dispersal routes for the ancestral populations of the two species according to the results of diyABC analyses. 

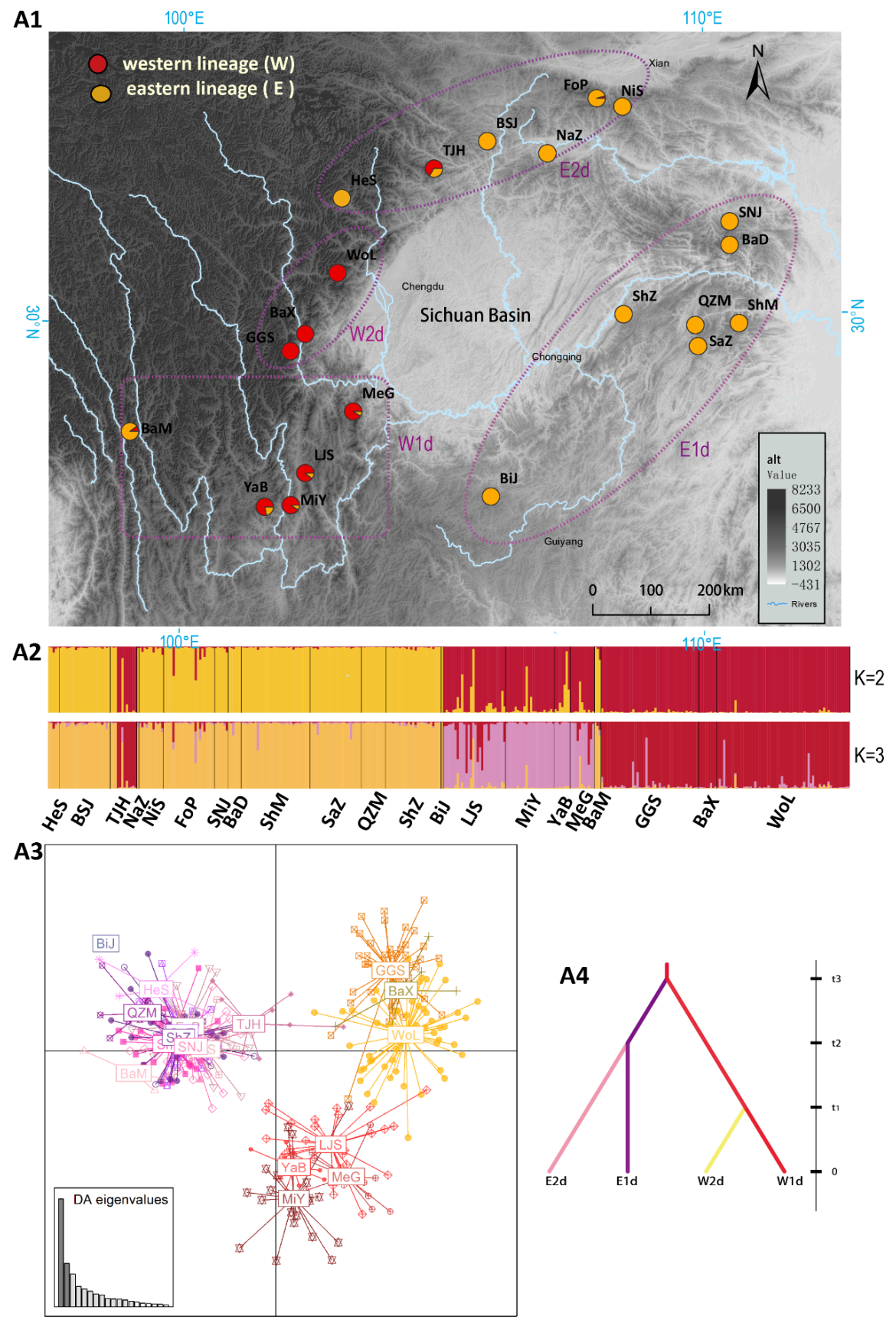

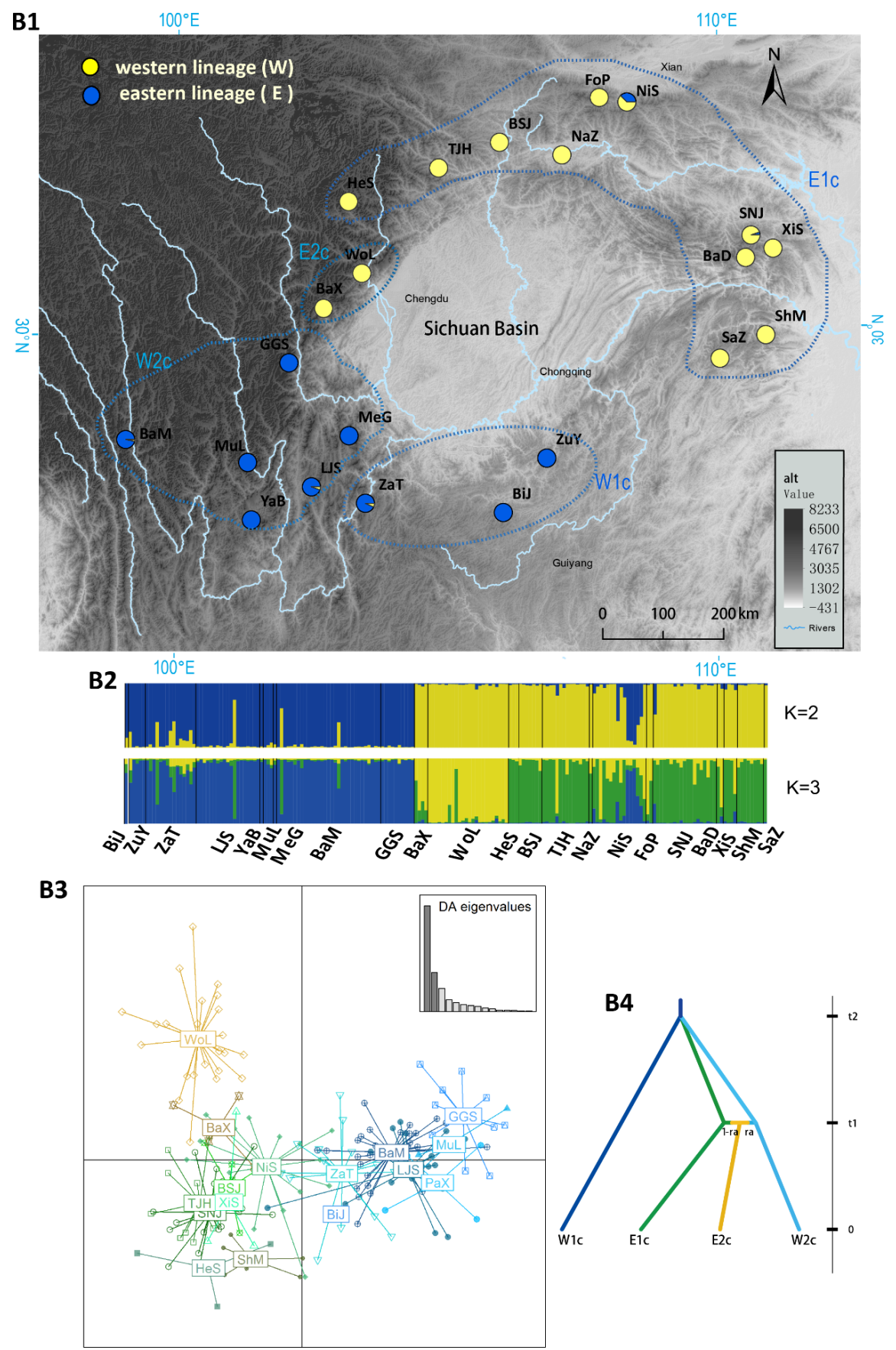

Fig. 4 Isolation-by-distance analysis (IBD) of $A$. draco(A) and A. chevrieri (B). (A1/B1) Adjusted 'ring distances', which assumed dispersal routes were following the ring configuration, were used. (A2/B2) 'Straight distances' between the sites, which may cross the uninhabitable basin, were used. 

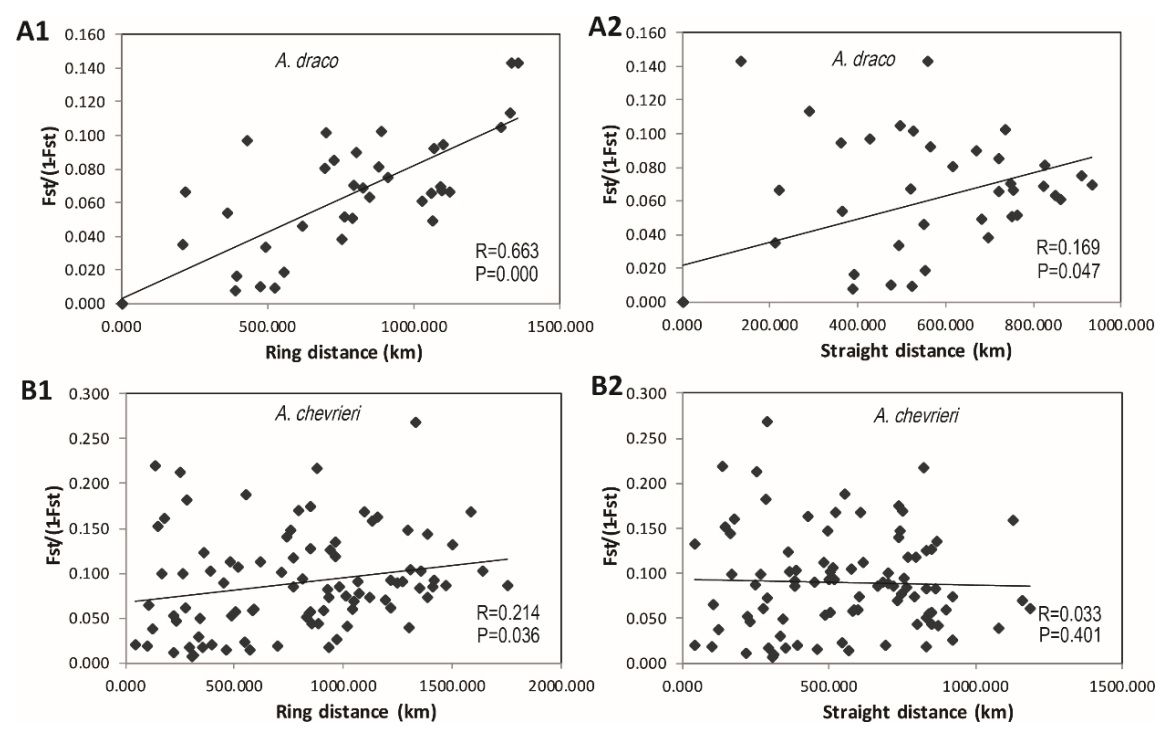

Fig. 5 Clinal analysis of $A$. draco (A) and A. chevrieri (B). The $\mathrm{x}$ axis represents the distance from site HeS (A) and GGS (B) following the potential suitable habitat around the Sichuan Basin. The y axis represents PC1 from the principal component analysis of the microsatellite dataset. 

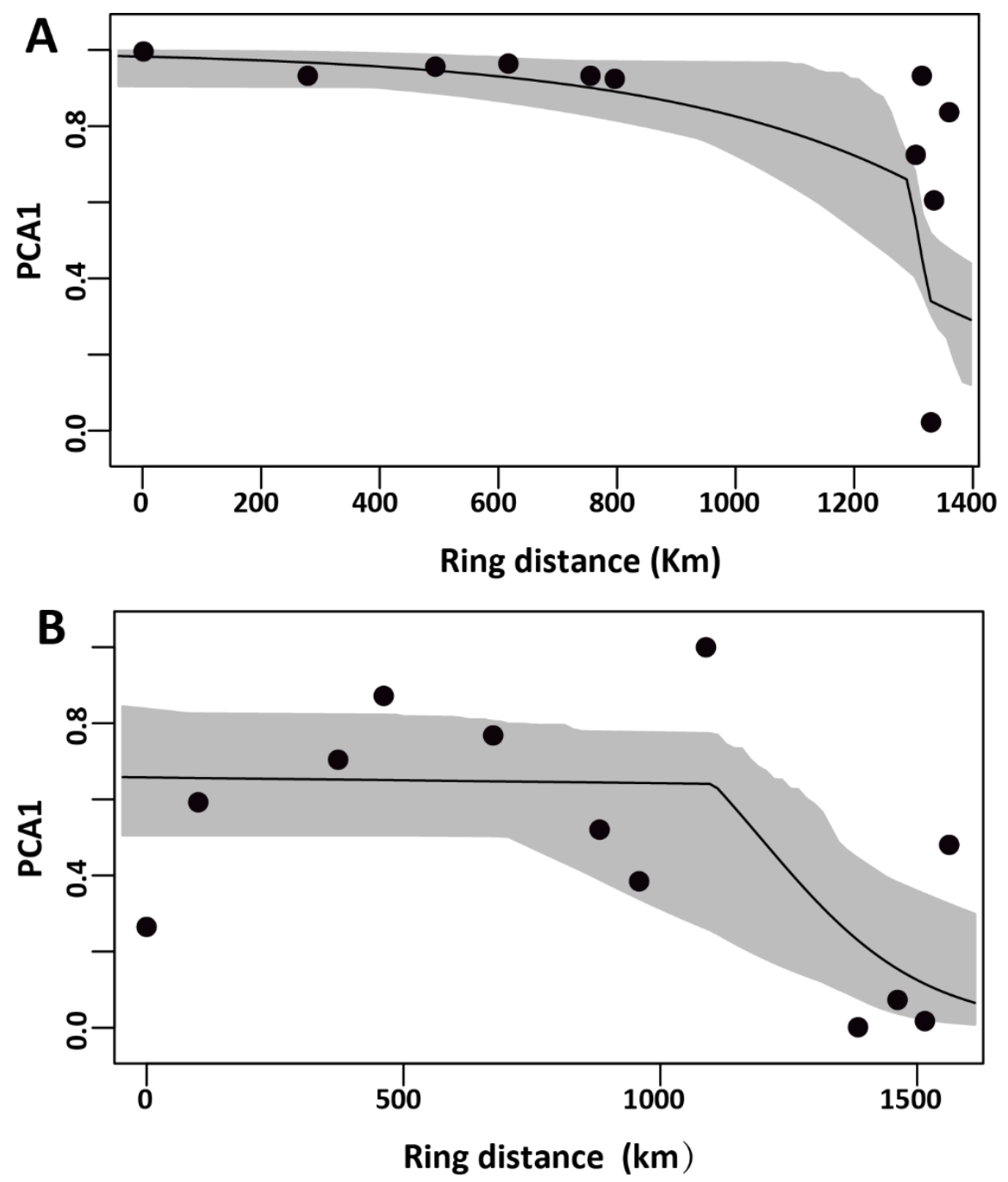

Fig. 6Redundancy analyses (RDA) of the contributions of environmental and spatial variables to the genetic variation of $A$. draco (A) and A. chevrieri (B). The graph shows the results of the model combining environmental and spatial variables as explanatory variables. MeanDiurRange: Mean Diurnal Range; TempSeas: Temperature Seasonality; AnnRange: Temperature Annual Range; PrecDryMonth: Precipitation of the driest month; PrecSeas: Precipitation Seasonality; PrecWarm: Precipitation of the Warmest Quarter; NPP: net primary productivity; PET: potential evapotranspiration. 

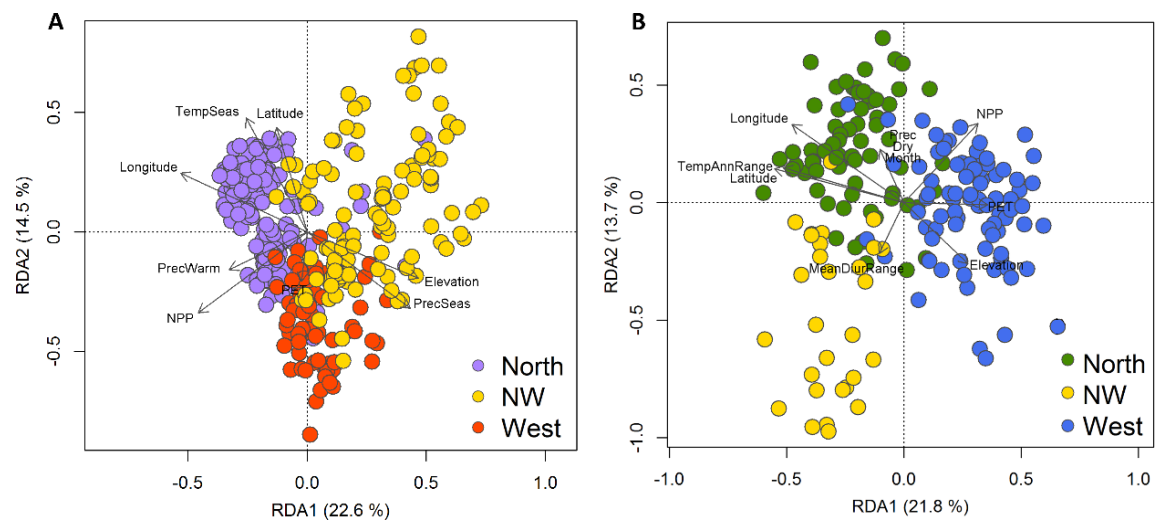

Fig. 7 Potential suitable distributional range of $A$. draco and $A$. chevrieri predicted by species distribution modeling (SDM). The distributional range at the last interglacial (LIG; approximately 130,000 BP), the last glacial maximum (LGM; approximately 22,000 BP) and present time were predicted. The scale on the left indicates the probability of occurrence.

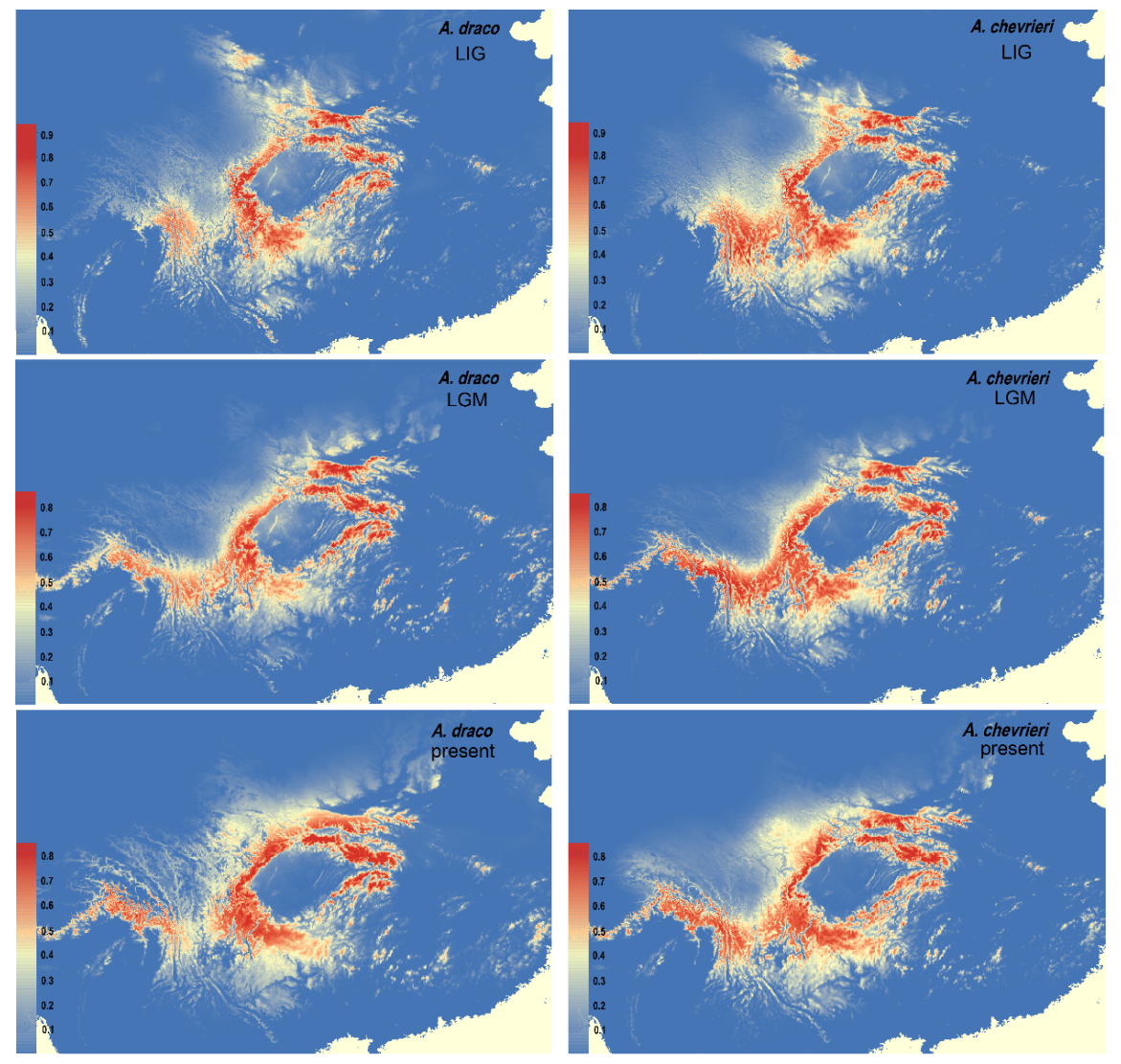

Table 1Redundancy analyses (RDA) of the contributions of environmental and spatial variables to the genetic variation of $A$. draco and A. chevrieri .

\begin{tabular}{lllll}
\hline A. draco & A. draco & A. chevrieri & A. chevrieri \\
\hline $\mathrm{R}^{2} a d j$ & $\mathrm{P}$ value & $\mathrm{R}^{2}$ adj & $\mathrm{P}$ value
\end{tabular}




\begin{tabular}{lllll}
\hline & A. draco & A. draco & A. chevrieri & A. chevrieri \\
\hline (a) Climatic + Spatial & 36.5 & $<0.001$ & 38.4 & $<0.001$ \\
(b) Climatic (spatial controlled) & 15.4 & $<0.001$ & 16.1 & $<0.001$ \\
(c) Spatial (climatic controlled) & 0.97 & $<0.001$ & 6.3 & $<0.001$ \\
\hline
\end{tabular}

Note : (a) the model combining environmental and spatial variables as explanatory variables; (b) the model with the isolated effect of environmental variables controlling for spatial influence; (c) the model with the isolated effect of spatial structure controlling for environmental influence. $\mathrm{R}^{2}$ adj : Adjusted R-Square. 